University of Wollongong

Research Online

Australian Institute for Innovative Materials -

Papers

Australian Institute for Innovative Materials

$1-1-2017$

\title{
Tuning the magnetic and structural transitions in TbCo2Mnx compounds
}

Chunsheng Fang

University of Wollongong, cf904@uowmail.edu.au

Jian Li Wang

University of Wollongong, Jilin University, University of New South Wales, jianli@uow.edu.au

Fang Hong

University of Wollongong

Wayne D. Hutchison

University of New South Wales

M. F Md Din

University of Wollongong

See next page for additional authors

Follow this and additional works at: https://ro.uow.edu.au/aiimpapers

Part of the Engineering Commons, and the Physical Sciences and Mathematics Commons

Research Online is the open access institutional repository for the University of Wollongong. For further information contact the UOW Library: research-pubs@uow.edu.au 


\title{
Tuning the magnetic and structural transitions in TbCo2Mnx compounds
}

\begin{abstract}
The wide ranging magnetic behavior in intermetallic compounds continues to attract broad interest. Effective control of their magnetic properties is of great importance for fundamental research and potential applications. In this work the structural and magnetic properties of TbCo2Mnx compounds are studied by a combination of temperature dependent synchrotron x-ray diffraction, neutron powder diffraction, specific heat, and magnetic measurements. Magnetization measurements show that the addition of $\mathrm{Mn}$ can modify the magnetic behavior significantly: first, the magnetic transition temperatures increase from $\sim 227 \mathrm{~K}$ to $332 \mathrm{~K}$ with $\mathrm{x}=0.0$ to 0.3 ; secondly, the nature of the magnetic transitions change from the first order to second order, as identified by three methods (Banerjee criterion, master curves of magnetic entropy changes, and detailed crystal structure analysis through neutron diffraction). Both synchrotron $\mathrm{x}$-ray diffraction and neutron diffraction confirm that a structural transition, from $\mathrm{cubic}^{\mathrm{Fd}} 3^{-} \mathrm{m}$ to rhombohedral $\mathrm{R}^{-} \mathrm{m}$ on cooling, occurred accompanying the magnetic transition. To further clarify the nature of the second order magnetic phase transitions, we have carried out a detailed critical exponent analysis. The derived critical exponents are close to the theoretical prediction from the mean-field model, indicating the magnetic interactions are long range. This work benefits our general understanding of magnetic interactions in intermetallic compounds and provides guidance to design a functional magnetic material for room temperature magnetic devices.
\end{abstract}

\section{Disciplines}

Engineering | Physical Sciences and Mathematics

\section{Publication Details}

Fang, C., Wang, J., Hong, F., Hutchison, W. D., Din, M., Studer, A. J., Kimpton, J. A., Dou, S. \& Cheng, Z. (2017). Tuning the magnetic and structural transitions in TbCo2Mnx compounds. Physical Review B: Condensed Matter and Materials Physics, 96 (6), 064425-1-064425-11.

\section{Authors}

Chunsheng Fang, Jian Li Wang, Fang Hong, Wayne D. Hutchison, M. F Md Din, Andrew J. Studer, Justin A. Kimpton, Shi Xue Dou, and Zhenxiang Cheng 


\title{
Tuning the magnetic and structural transitions in $\mathrm{TbCo}_{2} \mathrm{Mn}_{x}$ compounds
}

\author{
Chunsheng Fang, ${ }^{1}$ Jianli Wang,,${ }^{1,2,3,{ }^{*}}$ Fang Hong, ${ }^{1}$ Wayne D. Hutchison, ${ }^{3}$ M. F. Md Din, ${ }^{1}$ A. J. Studer, ${ }^{4}$ J. A. Kimpton, ${ }^{5}$ \\ Shixue Dou, ${ }^{1}$ and Zhenxiang Cheng ${ }^{1, \dagger}$ \\ ${ }^{1}$ Institute for Superconducting and Electronic Materials, Innovation Campus, University of Wollongong, \\ Squires Way, North Wollongong, NSW 2500, Australia \\ ${ }^{2}$ College of Physics, Jilin University, Changchun 130012, China \\ ${ }^{3}$ School of Physical, Environmental and Mathematical Sciences, The University of New South Wales, Canberra, ACT, 2600, Australia \\ ${ }^{4}$ Australian Nuclear Science and Technology Organisation (ANSTO), Lucas Heights, New South Wales 2234, Australia \\ ${ }^{5}$ Australian Synchrotron, 800 Blackburn Rd., Clayton 3168, Australia
}

(Received 10 May 2017; published 18 August 2017)

\begin{abstract}
The wide ranging magnetic behavior in intermetallic compounds continues to attract broad interest. Effective control of their magnetic properties is of great importance for fundamental research and potential applications. In this work the structural and magnetic properties of $\mathrm{TbCo}_{2} \mathrm{Mn}_{x}$ compounds are studied by a combination of temperature dependent synchrotron $\mathrm{x}$-ray diffraction, neutron powder diffraction, specific heat, and magnetic measurements. Magnetization measurements show that the addition of Mn can modify the magnetic behavior significantly: first, the magnetic transition temperatures increase from $\sim 227 \mathrm{~K}$ to $332 \mathrm{~K}$ with $x=0.0$ to 0.3 ; secondly, the nature of the magnetic transitions change from the first order to second order, as identified by three methods (Banerjee criterion, master curves of magnetic entropy changes, and detailed crystal structure analysis through neutron diffraction). Both synchrotron x-ray diffraction and neutron diffraction confirm that a structural transition, from cubic $F d \overline{3} m$ to rhombohedral $R \overline{3} m$ on cooling, occurred accompanying the magnetic transition. To further clarify the nature of the second order magnetic phase transitions, we have carried out a detailed critical exponent analysis. The derived critical exponents are close to the theoretical prediction from the mean-field model, indicating the magnetic interactions are long range. This work benefits our general understanding of magnetic interactions in intermetallic compounds and provides guidance to design a functional magnetic material for room temperature magnetic devices.
\end{abstract}

DOI: 10.1103/PhysRevB.96.064425

\section{INTRODUCTION}

The cubic Laves phases $R T_{2}(R=$ rare earth, $T=$ transition metal) series magnetic materials were studied intensively because of their unique electronic and magnetic structures, which lead to many physical properties such as giant magnetocaloric effect, magnetoresisitance [1,2], and hydrogen storage capacity [3]. Typical Laves phases include the cubic $\mathrm{C}_{15}\left(\mathrm{MgCu}_{2}\right)$, hexagonal $\mathrm{C}_{14}\left(\mathrm{MgZn}_{2}\right)$, and hexagonal $\mathrm{C}_{36}\left(\mathrm{MgNi}_{2}\right)$ [3]. They are ideal magnetic systems in which to study the effect of atomic occupation and $f-d$ orbital electron localization on magnetic and electronic properties [4].

Wang et al. [5,6] discovered $R \mathrm{Ni}_{2} \mathrm{Mn}(R=\mathrm{Tb}$, Dy, Ho, Er) compounds, which crystallize in the cubic $\mathrm{MgCu}_{2}$-type structure ( $F d \overline{3} m$, space group). Recently, it was reported that $R \mathrm{Co}_{2} \mathrm{Mn}$ with $R=$ Ho and Er also crystallize in the cubic $\mathrm{MgCu}_{2}$-type structure [2]. It was found that the introduction of Mn into $R T_{2}$ ( $T$ is $3 d$ or $4 d$ transition metal) compounds led to a significant modification of magnetic properties such as the Curie temperature [2,4,7-11], magnetic entropy change [4], saturation moment [2], the nature of the magnetic transition [4], and so on. It is accepted that the Curie temperature can be raised significantly with Mn doping due to the enhanced $3 d$ transition-metal ion interactions [5,6]. It is of interest that the $R \mathrm{~T}_{2} \mathrm{Mn}$ compounds were isostructural to $R T_{2}$ with the

\footnotetext{
*jianli@uow.edu.au

†cheng@uow.edu.au
}

$\mathrm{MgCu}_{2}$-type structure, although the ratio of the $R$ to $T$ is $1: 3$ [2,4,7-11], and the Mn was observed to occupy both the $R$ and $T$ sites. All the above modified magnetic properties and crystal structure inspired more work on related series materials such as $\mathrm{ErCo}_{2} \mathrm{Mn}$ [2], $\mathrm{HoNi}_{2} \mathrm{Mn}$ [9], $\mathrm{DyNi}_{2} \mathrm{Mn}[5,6]$, and $\mathrm{TbNi}_{2} \mathrm{Mn}$ $[6,7]$.

However, there is little systematic research specifically on the $\mathrm{RT}_{2} \mathrm{Mn}_{x}$ ( $x$ up to 1.0) series since the crystal structure is unstable when significant $\mathrm{Mn}$ is added. For example, $\mathrm{GdNi}_{2} \mathrm{Mn}_{x}(0 \leqslant x \leqslant 0.6)$ alloys were successfully synthesized and characterized by XRD. When the Mn concentration increases to a certain level $(x \geqslant 0.35)$, a second phase with rhombohedral $\mathrm{PuNi}_{3}$-type $(R 3 m)$ structure starts to form [10]. Recently, the investigation on $\mathrm{ErCo}_{2} \mathrm{Mn}_{x}$ ( $x=0$ to 0.8 ) also found that, when $x \leqslant 0.8$, the alloys are almost single phase $\left(\mathrm{MgCu}_{2}\right.$-type Laves-phase structure), while a second phase with cubic $\mathrm{Th}_{6} \mathrm{Mn}_{23}$-type structure appears for larger $x$ values [11].

Previous investigations of the $\mathrm{TbCo}_{2}$ compound indicated that $\mathrm{TbCo}_{2}$ has the $\mathrm{MgCu}_{2}$-type structure and shows paramagnetic behavior at room temperature. When cooled to lower temperature, it exhibits a rhombohedral distortion around the Curie temperature $T_{C}(=240 \mathrm{~K}$ ) (from paramagnetism to ferrimagnetism). The $\mathrm{Tb}-\mathrm{Co}$ coupling is antiferromagnetic with a larger moment in $\mathrm{Tb}$ site and almost equal Co moments at the $3 b$ and $9 e$ sites. The nature of the magnetic transition (PM to $\mathrm{FM}$ ) in $\mathrm{TbCO}_{2}$ was indicated to be second order according to Arrott plots [9,12-15]. However, relying solely on Arrott plots to decide the nature of transition is not always prudent $[16,17]$. 


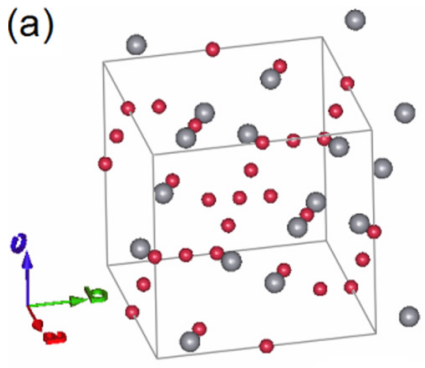

$\mathrm{Tb} \odot \mathrm{Co} \bullet$

(b)
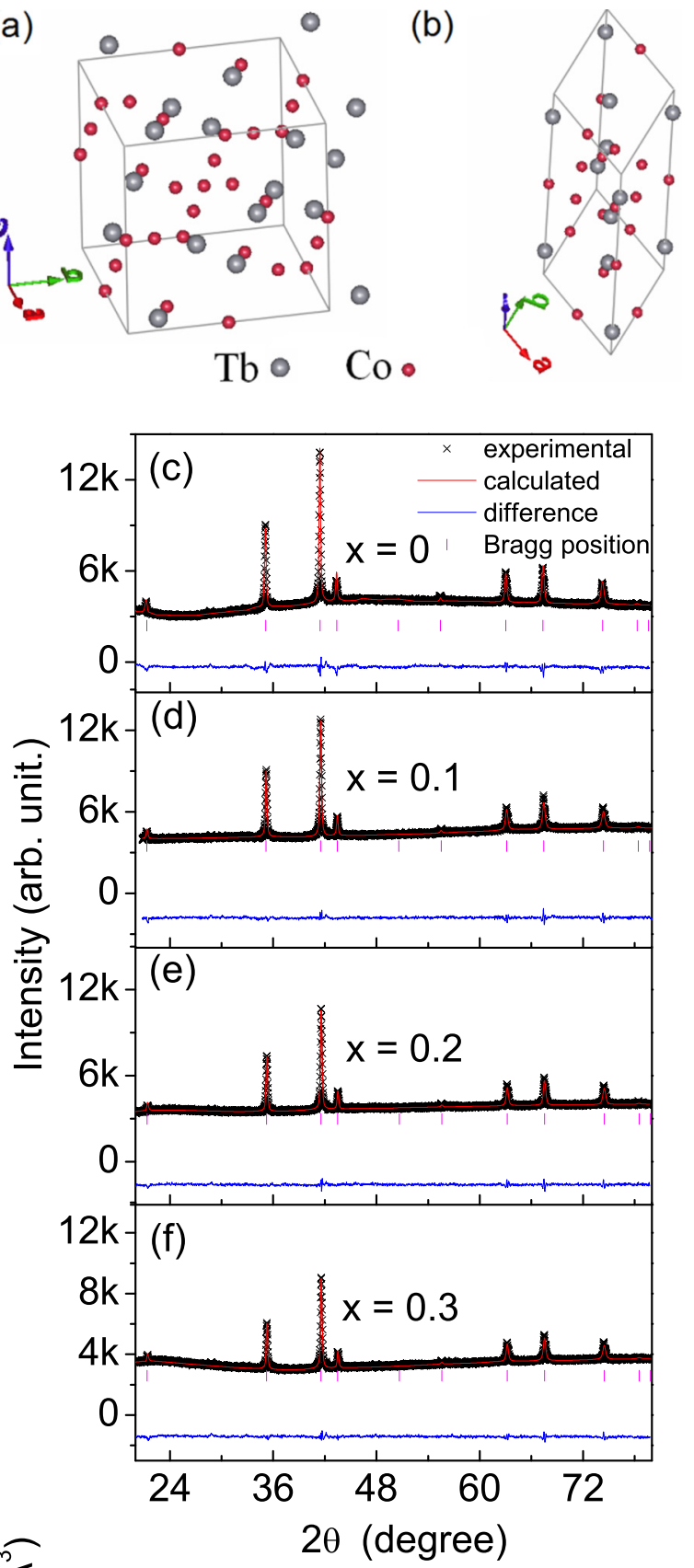

$\widetilde{2}$

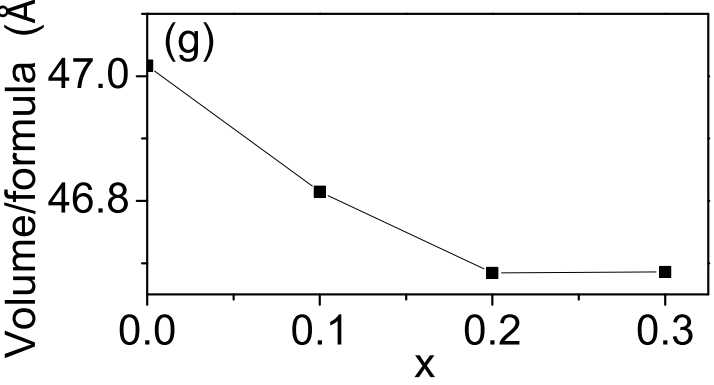

FIG. 1. Crystal structures with space group of (a) $F d \overline{3} m$ and (b) $R \overline{3} m$. (c), (d,) (e), and (f) are room temperature XRD refinements of $\mathrm{TbCo}_{2} \mathrm{Mn}_{x}(x=0,0.1,0.2$, and 0.3$)$, respectively. $(\mathrm{g})$ is the $x$ value dependence of the volume per chemical formula.

In this paper, we present a detailed investigation on structural and magnetic properties of $\mathrm{TbCo}_{2} \mathrm{Mn}_{x}(x=0,0.1$,
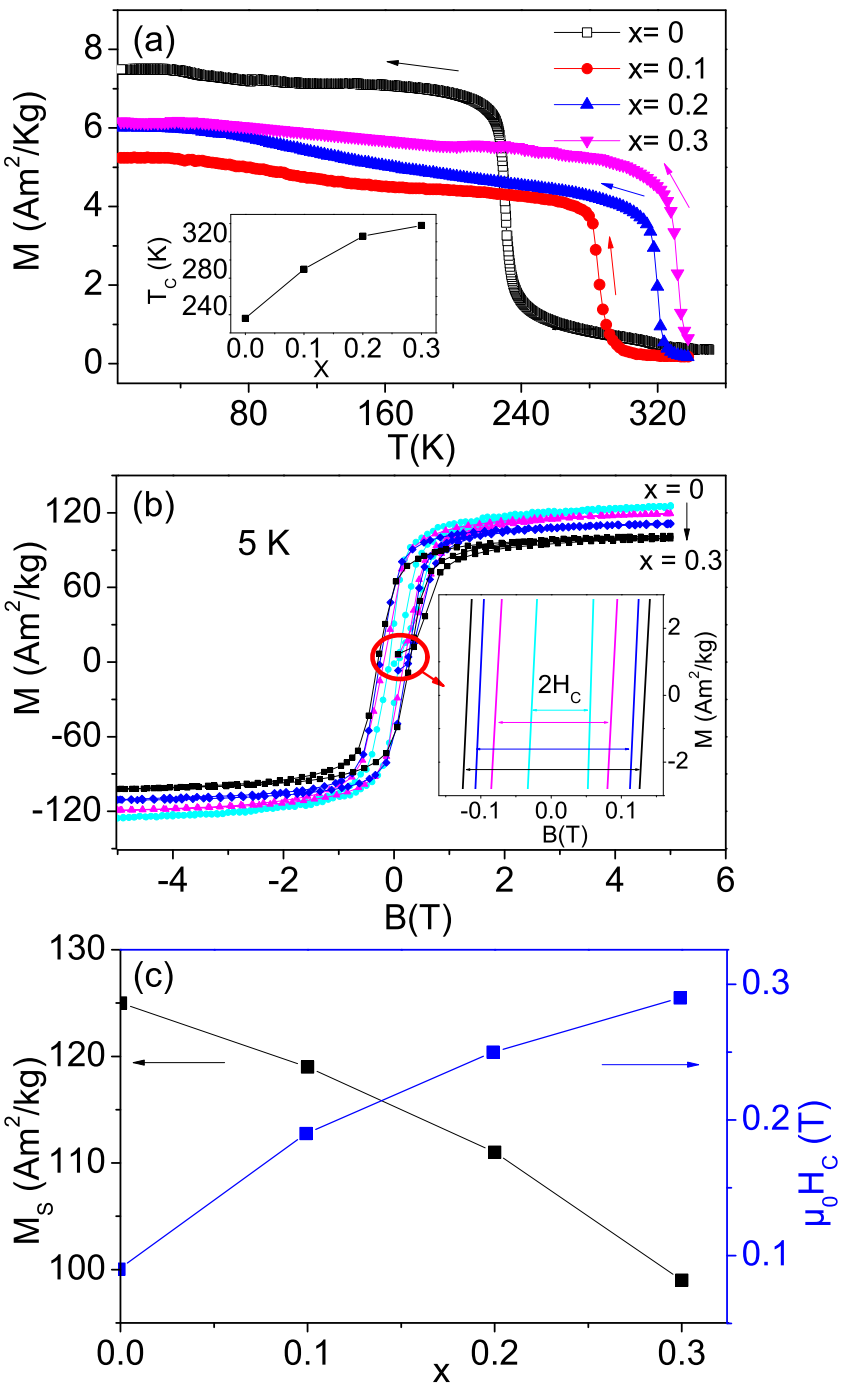

FIG. 2. (a) Temperature dependent magnetization with field cooling (FC at the field of $B=0.01 \mathrm{~T}$ for $\mathrm{TbCo}_{2} \mathrm{Mn}_{x}(x=0,0.1$, 0.2 , and 0.3). The inset shows the $x$ value dependence of the Curie temperature $T_{C}$. (b) Hysteresis loops at $5 \mathrm{~K}$ for $\mathrm{TbCo}_{2} \mathrm{Mn}_{x}(x=0$, $0.1,0.2$, and 0.3 ). The inset is an enlargement for small $B$ and $M$ values to show the coercivity $H_{C}$. (c) Plot of the dependence of saturation magnetization $M_{S}$ and coercivity $\mu_{0} H_{C}$ versus $x$ at $5 \mathrm{~K}$.

0.2 , and 0.3 ) compounds using variable temperature neutron diffraction and synchrotron x-ray diffraction. Analysis of the temperature dependent neutron diffraction data shows that the nature of magnetic transition in $\mathrm{TbCo}_{2}$ is first order with a sharp volume change near $T_{C}$. However, this first order magnetic phase transition for $x=0$ changes to second order after the introduction of $\mathrm{Mn}$. We plotted master curves of the magnetic entropy changes $[18,19]$ to clearly identify the transformation from first order to second order, as well as detailed refinement of diffraction data near the magnetic phase transition temperature to verify the change in crystal structure. Finally, the critical behavior around $T_{C}$ was investigated using the Kouvel-Fisher technique to precisely analyze the nature of the second order transitions for all the $\mathrm{TbCo}_{2} \mathrm{Mn}_{x}(x=0.1$, 0.2 , and 0.3 ) compounds. 


\section{EXPERIMENT}

The polycrystalline $\mathrm{TbCo}_{2} \mathrm{Mn}_{x}$ compounds with $x=0$, $0.1,0.2$, and 0.3 were prepared by arc melting the constituent elements of $99.9 \%$ purity under argon atmosphere. The values of $x$ referred to hereafter are these nominal ratios of the starting mixture materials. Note $3 \%$ additional Mn was added initially to compensate nonproportionate losses of Mn during the arcmelting and annealing process. We have evaluated the loss by measuring the weight of materials before and after preparation; the error in $x$ is less than 3\%. The prepared ingots were wrapped in tantalum foil and sealed in quartz glass tubes under vacuum and annealed at $1173 \mathrm{~K}$ for 7 days and then quenched into ice water. All the samples were checked with X-ray powder diffraction at room temperature using a PANalytical diffractometer with $\mathrm{Cu} K \alpha$ radiation. The dc magnetization measurements and heat capacity were performed using a Quantum Design physical properties measurement system (PPMS) from $5 \mathrm{~K}$ to $340 \mathrm{~K}$, and from $2 \mathrm{~K}$ to $320 \mathrm{~K}$, respectively. The magnetic and structural transitions were investigated via neutron diffraction experiments at the Wombat (high-intensity powder diffractometer) at the OPAL facility (Lucas Height, Australia) with a neutron wavelength of $2.4139 \AA$ from $5 \mathrm{~K}$ to $400 \mathrm{~K}$. The crystal structure transitions were also examined by the synchrotron X-ray powder diffraction at the Australian Synchrotron using a wavelength of $0.6884 \AA$ at temperatures from $90 \mathrm{~K}$ to $400 \mathrm{~K}$.

\section{RESULTS AND DISCUSSION}

\section{A. Crystal structure at room temperature}

Room temperature XRD was performed to check that all the samples were single phase. The Rietveld refinement confirmed that $\mathrm{TbCo}_{2} \mathrm{Mn}_{0.1}, \mathrm{TbCo}_{2} \mathrm{Mn}_{0.2}$, and $\mathrm{TbCo}_{2} \mathrm{Mn}_{0.3}$ all have the same crystal structure as the parent compound $\mathrm{TbCo}_{2}$ [cubic with $F d \overline{3} m$ space group shown in Fig. 1(a)]. We have adopted the similar refinement approach as described in Refs. [5,6] to assume that $\mathrm{Mn}$ atoms are located at both $\mathrm{Tb}$ and $\mathrm{Co}$

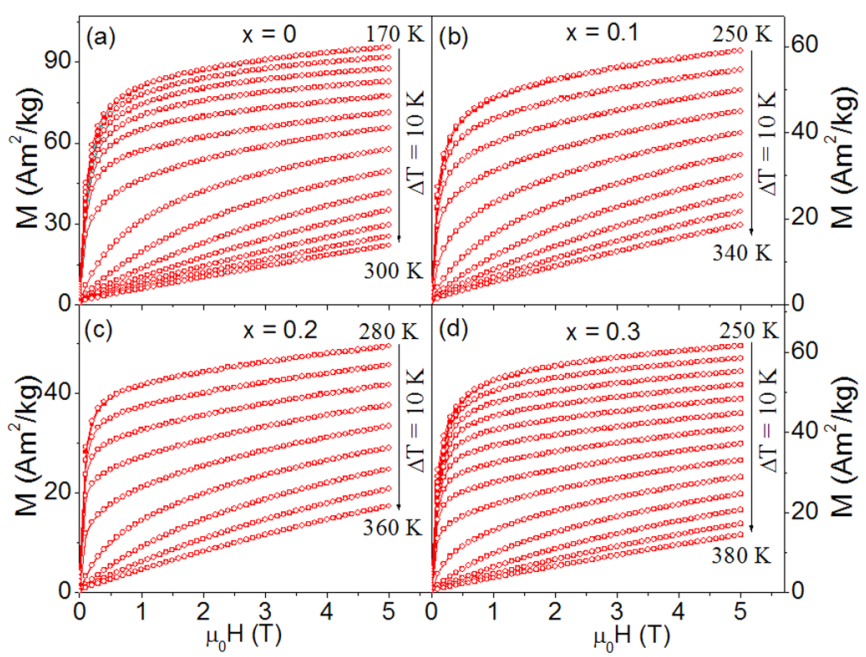

FIG. 3. Isothermal magnetization versus magnetic field at various temperatures in both increasing field (full red symbol) and decreasing field sweeps (hollow red symbols) for (a) $\mathrm{TbCo}_{2}$, (b) $\mathrm{TbCo}_{2} \mathrm{Mn}_{0.1}$, (c) $\mathrm{TbCo}_{2} \mathrm{Mn}_{0.2}$, and (d) $\mathrm{TbCo}_{2} \mathrm{Mn}_{0.3}$. sites with some vacancies at the $\mathrm{Tb}$ site in order to make $\mathrm{TbCo}_{2} \mathrm{Mn}_{x}$ able to adopt the $\mathrm{TbCo}_{2}$ structure. Here taking $\mathrm{TbCo}_{2} \mathrm{Mn}_{0.1}$ as an example, we have rewritten $\mathrm{TbCo}_{2} \mathrm{Mn}_{0.1}$ as $\left(\mathrm{Tb}_{1+v} \mathrm{Mn}_{y}\right)\left(\mathrm{Co}_{2} \mathrm{Mn}_{0.1-y}\right)$ and let $(1+v+y) /(2+0.1-y)$ equal 1:2 ( $v$ represent the number vacancy at the $\mathrm{Tb}$ site and $y$ stands for the number of $\mathrm{Mn}$ atoms occupying $\mathrm{Co}$ sites). In the case of the $\mathrm{TbCo}_{2} \mathrm{Mn}_{x}$ compounds it has been found that the best refinement has been obtained when $v=0.0$ which indicates that no vacancy exists in reality for $\mathrm{TbCo}_{2} \mathrm{Mn}_{x}$ compounds. The refinements of these XRD patterns at room temperature are shown in Fig. 1(c), Fig. 1(d), Fig. 1(e), and Fig. 1(f), respectively. The dependence of unit cell volume on the $x$ value is shown in Fig. $1(\mathrm{~g})$, from which it can be noted that the volume per chemical formula decreases from $47.02 \AA^{3}$ to $46.68 \AA^{3}$ as the $x$ value increases from 0 to 0.3 .

\section{B. Magnetic phase transition}

The magnetization versus temperature curves for $\mathrm{TbCo}_{2} \mathrm{Mn}_{x}(x=0,0.1,0.2$, and 0.3$)$ compounds are shown in

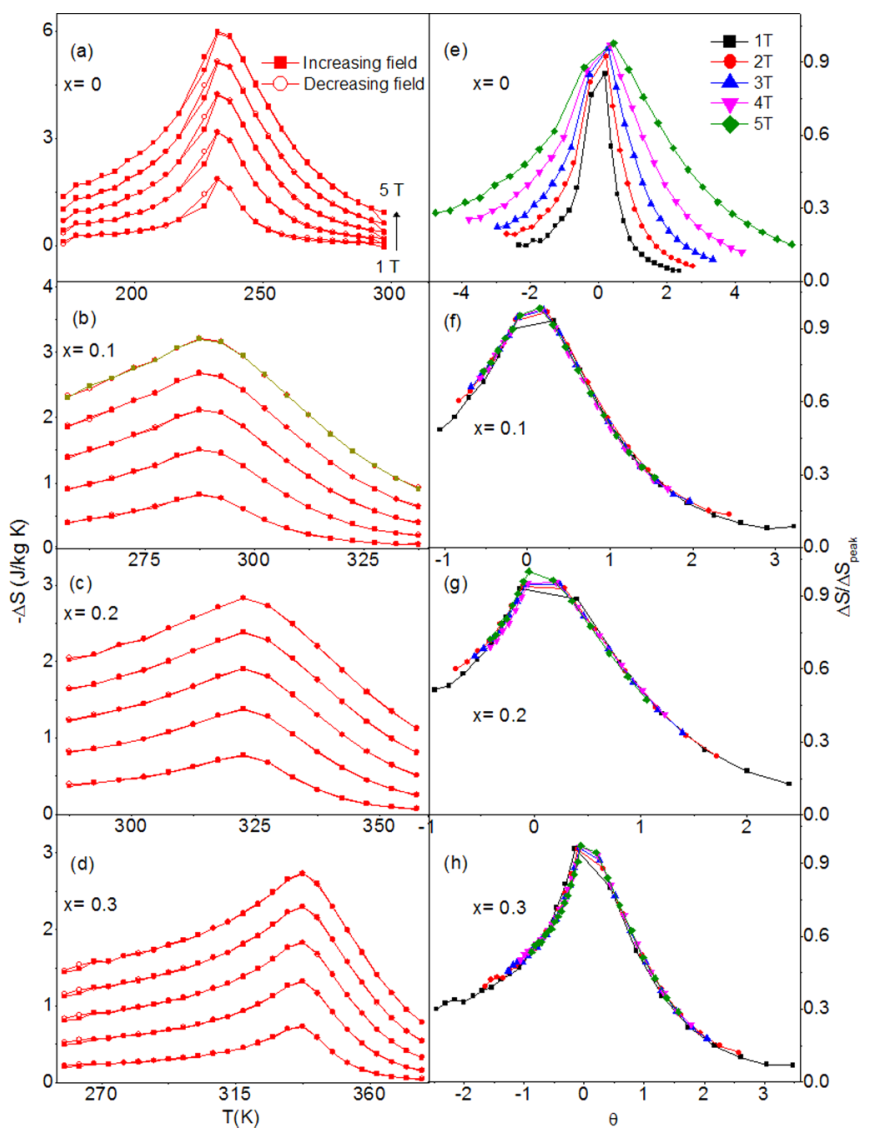

FIG. 4. Magnetic entropy changes for a range of temperatures and for magnetic field changes of $\mu_{o} \Delta H=1 \mathrm{~T}$ to $5 \mathrm{~T}$ [the curves corresponding to each field change are denoted by the arrow which shows the increasing order bottom to top in (a)] of (a) $\mathrm{TbCo}_{2}$, (b) $\mathrm{TbCo}_{2} \mathrm{Mn}_{0.1}$, (c) $\mathrm{TbCo}_{2} \mathrm{Mn}_{0.2}$, and (d) $\mathrm{TbCo}_{2} \mathrm{Mn}_{0.3}$. Increasing field data are represented by full red symbols and decreasing field data are open red symbols. The corresponding normalized magnetic entropy changes versus the rescaled temperature $\ominus$ are in (e), (f), (g), and (h), respectively. 
Fig. 2(a), measured with field cooling and at $B=0.01 \mathrm{~T}$. The Curie temperature $T_{C}$ is defined as the point where the slope of the temperature dependent $d M / d T$ curve is a minimum. It can be seen clearly that, with the $x$ value increasing from 0 to $0.3, T_{C}$ increases from $230 \mathrm{~K}$ to $284 \mathrm{~K}, 320 \mathrm{~K}$, and $332 \mathrm{~K}$, respectively [see inset of Fig. 2(a)]. The increase in $T_{C}$ with Mn concentration $x$ can be ascribed to enhanced exchange interactions between the $3 d$ transition metals $[8,20]$.

The magnetic hysteresis loops at $5 \mathrm{~K}$ for each sample are shown in Fig. 2(b). We can see directly that as Mn content increases the saturation magnetic moment $M_{S}$ decreases with increasing $x$ while the coercivity $H_{C}$ increases. From Fig. 2(c), it can be seen that the $M_{S}$ value decreases from $125 \mathrm{~A} \mathrm{~m}^{2} / \mathrm{kg}$ to $119 \mathrm{~A} \mathrm{~m}^{2} / \mathrm{kg}, 111 \mathrm{~A} \mathrm{~m}^{2} / \mathrm{kg}$, and $99 \mathrm{~A} \mathrm{~m}^{2} / \mathrm{kg}$, with the value of $x$ increasing from 0 to $0.1,0.2$, and 0.3 , respectively. It is well accepted in rare earth transition metal compounds that heavy rare earth moments couple antiferromagnetically with transition metal moments. If we assume here therefore that the Tb-sublattice moments also couple in an antiparallel fashion and are constant with increasing Mn moment, then the decrease in Ms with increasing $x$ indicates that the Mn atoms carry a magnetic moment in line with that concluded by the neutron diffraction analysis in Sec. VII. Meanwhile, the coercive field $H_{C}$ increases from $0.09 \mathrm{~T}$ to $0.19 \mathrm{~T}, 0.25 \mathrm{~T}$, and $0.29 \mathrm{~T}$ with $x$ value increasing from 0 to $0.1,0.2$, and 0.3 , respectively. The increased coercive fields suggest that the crystal symmetry of the compound was reduced, with anisotropy being enhanced after more Mn was introduced.

\section{Magnetocaloric effect}

The field dependencies of magnetization near the Curie temperatures are shown in Figs. 3(a), 3(b), 3(c), and 3(d) for $x=0,0.1,0.2$, and 0.3 , respectively. Consistent with the $M-T$ curves of Fig. 2(a), the magnetic FM-PM transition also can be detected in the magnetization-field $(M-H)$ isotherms in Figs. 3(a), 3(b), 3(c), and 3(d): with the temperature increasing near the Curie temperature, the $M-H$ isotherms transform

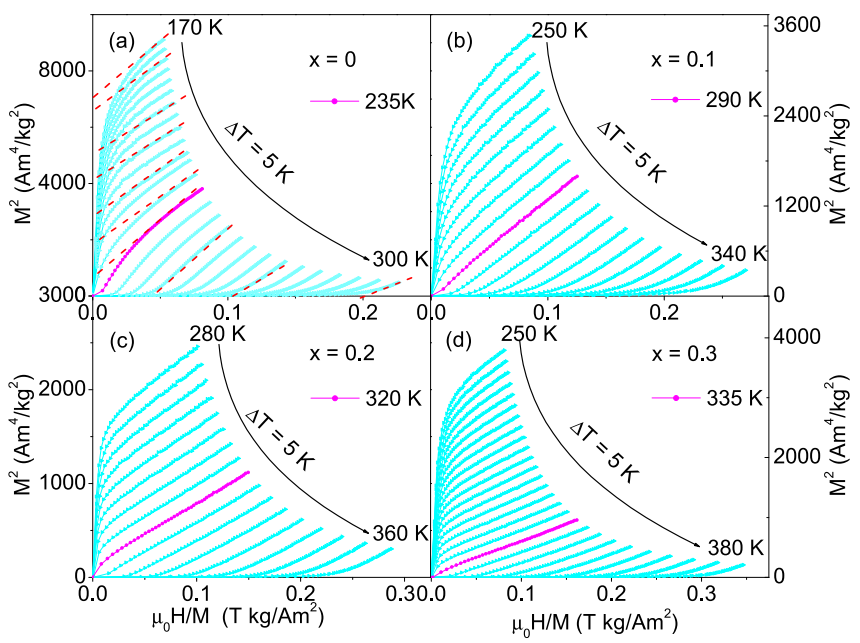

FIG. 5. Arrott plots: isotherms of $M^{2}$ vs $H / M$ for decreasing field at different temperatures around $T_{C}$ of (a) $\mathrm{TbCo}_{2}$, (b) $\mathrm{TbCo}_{2} \mathrm{Mn}_{0.1}$, (c) $\mathrm{TbCo}_{2} \mathrm{Mn}_{0.2}$, and (d) $\mathrm{TbCo}_{2} \mathrm{Mn}_{0.3}$. The magenta curves are the Arrott plots near the Curie temperature for each sample. from curved hysteresis loops to overlapping straight lines. Moreover, it can be seen that the hysteresis loss for all the series samples $(x=0,0.1,0.2$, and 0.3$)$ are negligible. The latter is desirable for increased magnetic refrigeration working efficiency for the materials [8].

To determine the magnetic entropy changes $\Delta S_{M}$ from the isothermal magnetization curves, the standard Maxwell relationship was used as follows:

$$
\Delta S_{M}(T, B)=\int_{0}^{B^{\max }}\left(\frac{\partial M(B, T)}{\partial T}\right)_{B} d B .
$$

The calculated temperature dependent magnetic entropy changes for the $\mathrm{TbCo}_{2} \mathrm{Mn}_{x}(x=0,0.1,0.2$, and 0.3$)$ samples
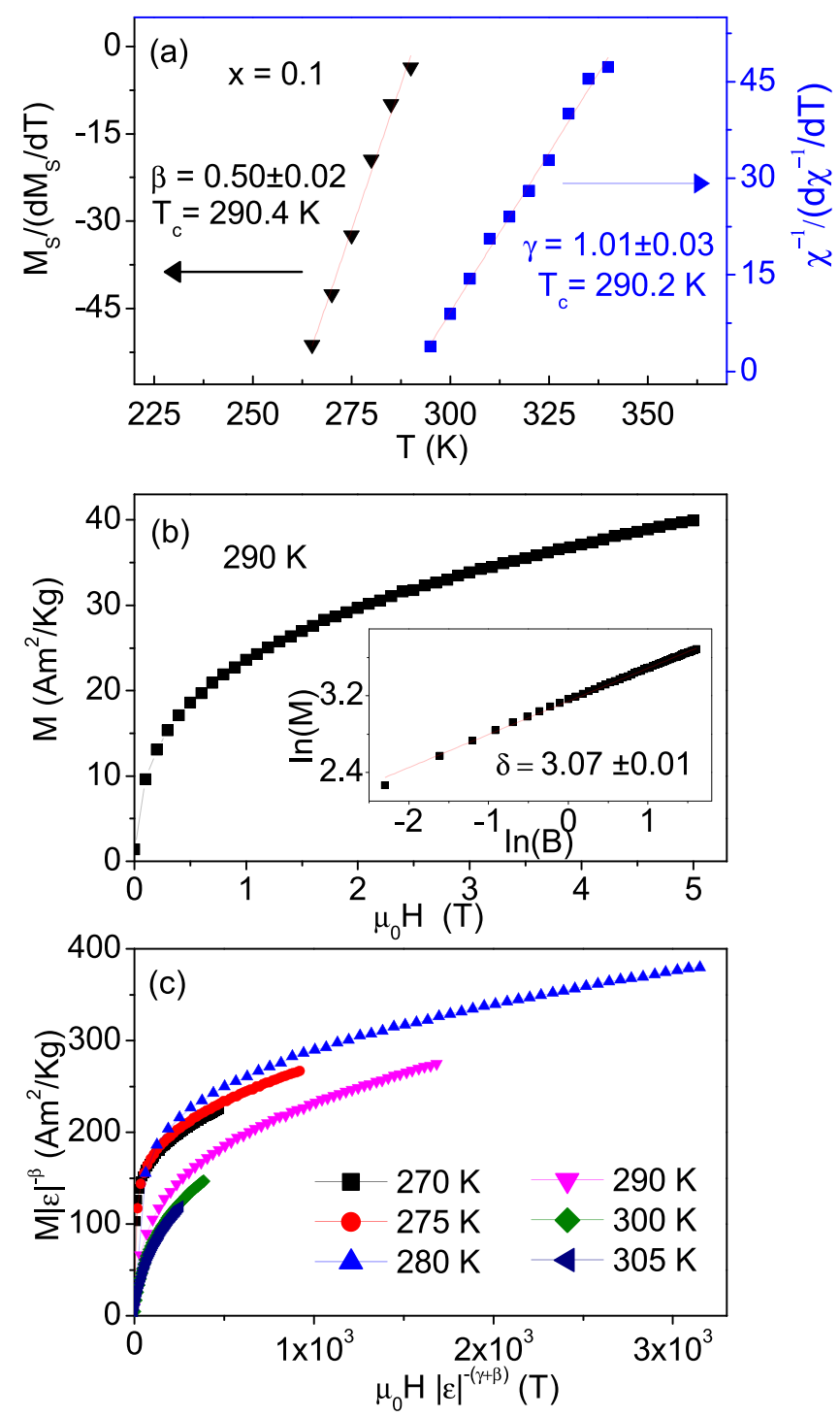

FIG. 6. Critical exponent analysis of $\mathrm{TbCo}_{2} \mathrm{Mn}_{0.1}$ for (a) KouvelFisher plot for the spontaneous magnetization $M_{S}(T)$ and the inverse initial susceptibility $\chi^{-1}$ [solid lines are fitted using Eqs. (3) and (4)]. (b) Critical isotherm of $M$ vs $H$ close to the Curie temperature $T_{c}=290 \mathrm{~K}$. Inset shows the same data on a log-log scale and the straight line is the linear fit following Eq. (5). The critical exponent $\delta$ is obtained from the slope of the linear fit. (c) Scaling plots indicating universal curves below and above $T_{C}$. 

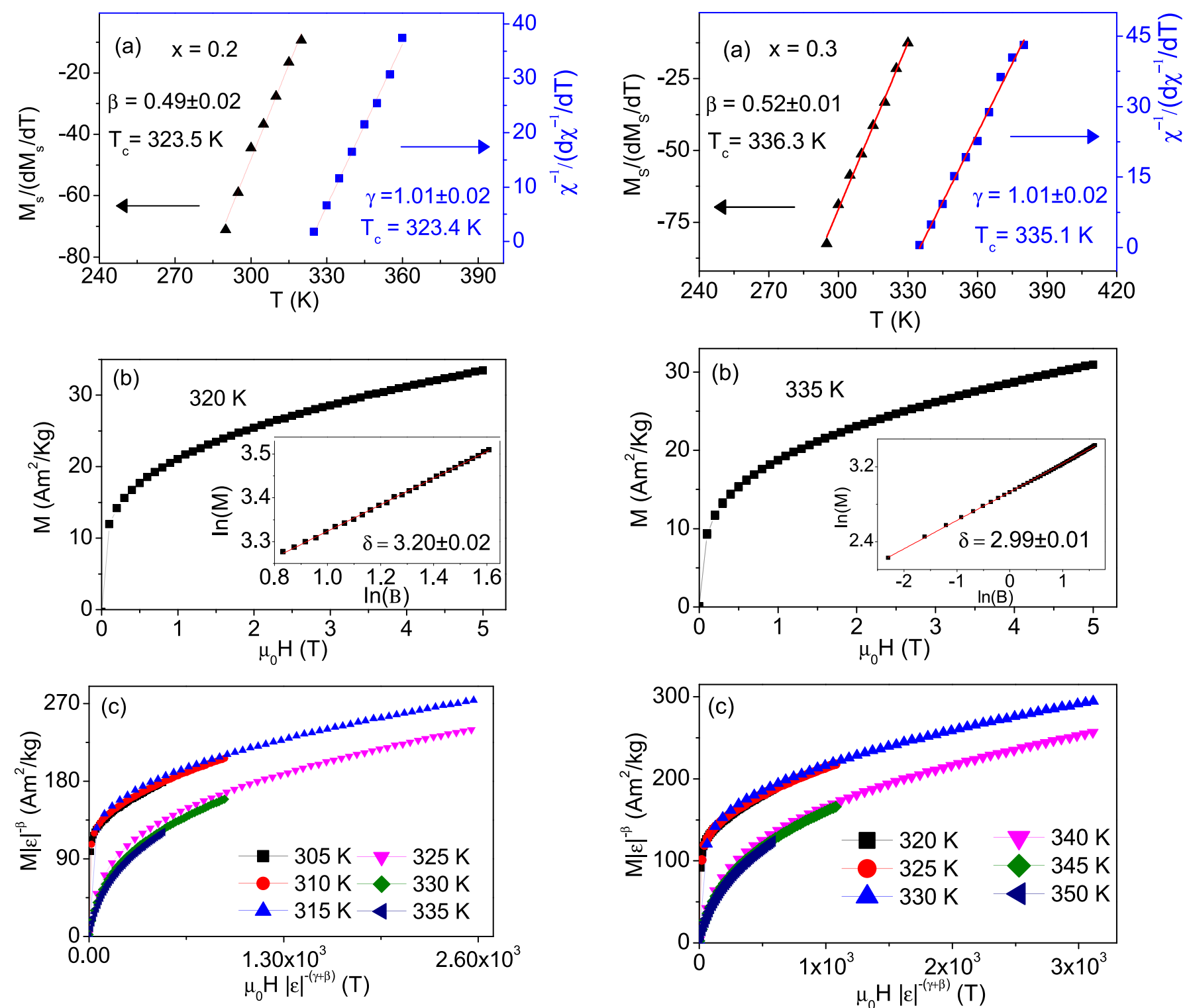

FIG. 7. Critical exponent analysis of $\mathrm{TbCo}_{2} \mathrm{Mn}_{0.2}$ for (a) KouvelFisher plot for the spontaneous magnetization $M_{S}(T)$ and the inverse initial susceptibility $\chi^{-1}$ [solid lines are fitted using Eqs. (3) and (4)]. (b) Critical isotherm of $M$ vs $H$ close to the Curie temperature $T_{c}=320 \mathrm{~K}$. Inset shows the same on log-log scale and the straight line is the linear fit following Eq. (5). The critical exponent $\delta$ is obtained from the slope of the linear fit. (c) Scaling plots indicating universal curves below and above $T_{C}$.

for both ramp up and ramp down with field changes from $0 \mathrm{~T}$ to $5 \mathrm{~T}$ are shown in Figs. 4(a), 4(b), 4(c), and 4(d).

From Fig. 4, it can be seen that for each sample there is a peak of magnetic entropy change $-\Delta S^{\max }$ near the Curie temperature for each magnetic field change ( $0 \mathrm{~T}$ to $5 \mathrm{~T})$ associated with the magnetic transition from the paramagnetic to the ferromagnetic state. Comparing the peak values, it is found that $\mathrm{TbCo}_{2}$ has the largest $-\Delta S^{\max }$ value of $6.0 \mathrm{~J} \mathrm{~kg}^{-1} \mathrm{~K}^{-1}$ followed by $3.2 \mathrm{~J} \mathrm{~kg}^{-1} \mathrm{~K}^{-1}, 2.83 \mathrm{~J} \mathrm{~kg}^{-1} \mathrm{~K}^{-1}$, and $2.74 \mathrm{~J} \mathrm{~kg}^{-1} \mathrm{~K}^{-1}$ for $\mathrm{TbCo}_{2} \mathrm{Mn}_{0.1}, \mathrm{TbCo}_{2} \mathrm{Mn}_{0.2}$, and $\mathrm{TbCo}_{2} \mathrm{Mn}_{0.3}$, respectively. The differences in the magnetic entropy for these compounds reflect that the type of magnetic transition has changed from

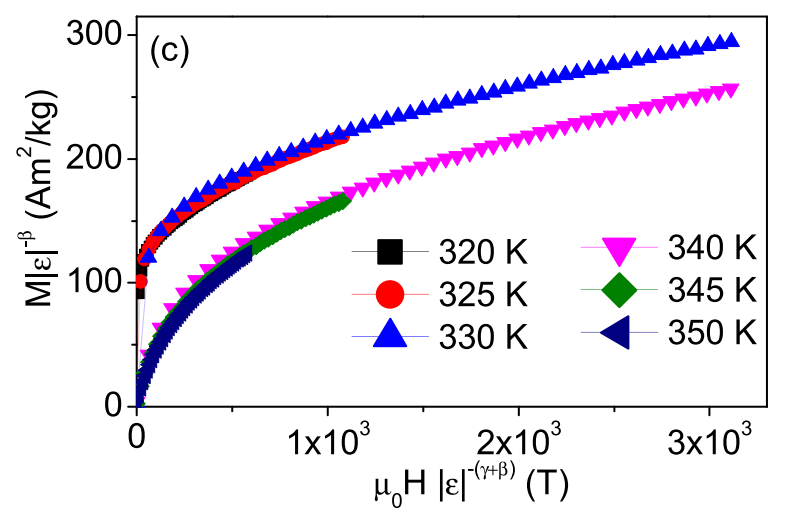

FIG. 8. Critical exponent analysis of $\mathrm{TbCo}_{2} \mathrm{Mn}_{0.3}$ for (a) KouvelFisher plot for the spontaneous magnetization $M_{S}(T)$ and the inverse initial susceptibility $\chi^{-1}$ [solid lines are fitted using Eqs. (3) and (4)]. (b) Critical isotherm of $M$ vs $H$ close to the Curie temperature $T_{c}=335 \mathrm{~K}$. Inset shows the same on $\log -\log$ scale and the straight line is the linear fit following Eq. (5). The critical exponent $\delta$ is obtained from the slope of the linear fit. (c) Scaling plots indicating universal curves below and above $T_{C}$.

the first order for $\mathrm{TbCo}_{2}$ to second order for $\mathrm{TbCo}_{2} \mathrm{Mn}_{x}$ $(x=0.1,0.2$, and 0.3$)$. Furthermore, as additional $\mathrm{Mn}$ is added the value of the $-\Delta S^{\max }$ decreases indicating that the contribution of structure entropy (i.e., magnetic volume effect) continues to weaken. The detailed volume change can be seen in Figs. 11-14.

Based on the magnetic entropy change of Figs. 4(a)-4(d), normalized plots of the magnetic entropy changes as a function of the rescaled temperature curves $[18,19]$ can be deduced. and these are plotted in Figs. 4(e)-4(h). The normalization of the magnetic entropy changes was defined as the value of $\Delta S / \Delta S_{\text {peak }}$ for each magnetic field change $\mu_{0} \Delta H$, while the 


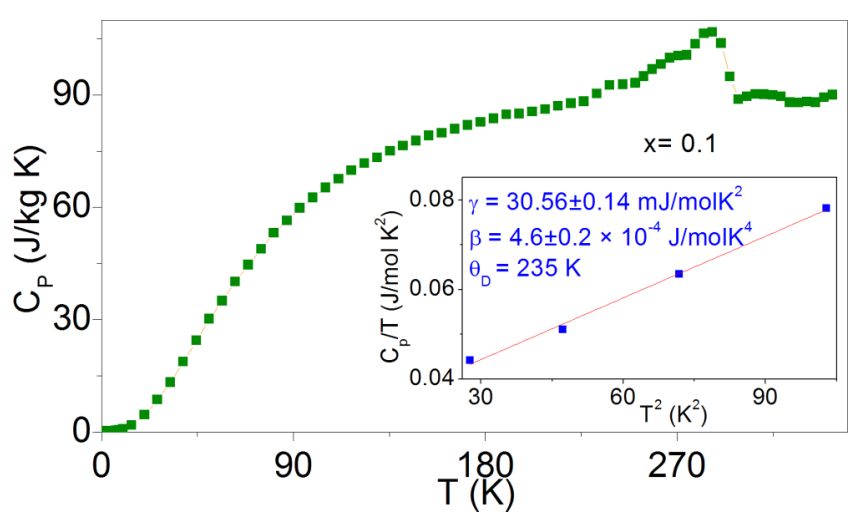

FIG. 9. Heat capacity vs temperature curve of $\mathrm{TbCo}_{2} \mathrm{Mn}_{0.1}$ at $B=0 \mathrm{~T}$ and inset shows $C_{p} / T$ vs $T^{2}\left(K^{2}\right)$ straight line with linear fitting.

rescaled temperature $\ominus$ was specified as follows $[18,19]$ :

$$
\theta=\left\{\begin{array}{l}
\left(T_{\text {peak }}-T\right) /\left(T_{r 1}-T_{\text {peak }}\right), \\
\left(T-T_{\text {peak }}\right) /\left(T_{r 2}-T_{\text {peak }}\right),
\end{array}\right.
$$

where $T_{r 1}$ and $T_{r 2}$ is the temperature point where there is half maximum value of the peak magnetic entropy change, respectively $\left(T_{r 2}<T_{\text {peak }}<T_{r 1}\right)$. In turn these curves can be used to indicate the nature of the magnetic transitions. From Figs. 4(e) to 4(h), it is very obvious that the entropy curves of the $x=0$ sample are highly dispersed for different magnetic field changes, while all the Mn doped sample normalization curves are overlapping for different fields. This is strong evidence suggesting the introduction of Mn does change the nature of the magnetic transition from the first order to the second order.

\section{Critical behavior at the magnetic phase transition temperature}

From Fig. 5(a) for $\mathrm{TbCo}_{2}$, it can be found that an " $s$ " shaped curve appears in the Arrott plot of $M^{2}$ vs $B / M$ at $235 \mathrm{~K}$, indicating that the magnetic transition of $\mathrm{TbCo}_{2}$ is a first order transition. While for Figs. 5(b), 5(c), and 5(d), only positive slopes are present, so it can be concluded that all transitions in these compounds are second order. The implication of this observation is that the addition of $\mathrm{Mn}$ into $\mathrm{TbCo}_{2}$ leads to a change of magnetic transition order from first order to second order.

In order to get a better understanding of the critical behaviors around the transition temperatures in these compounds
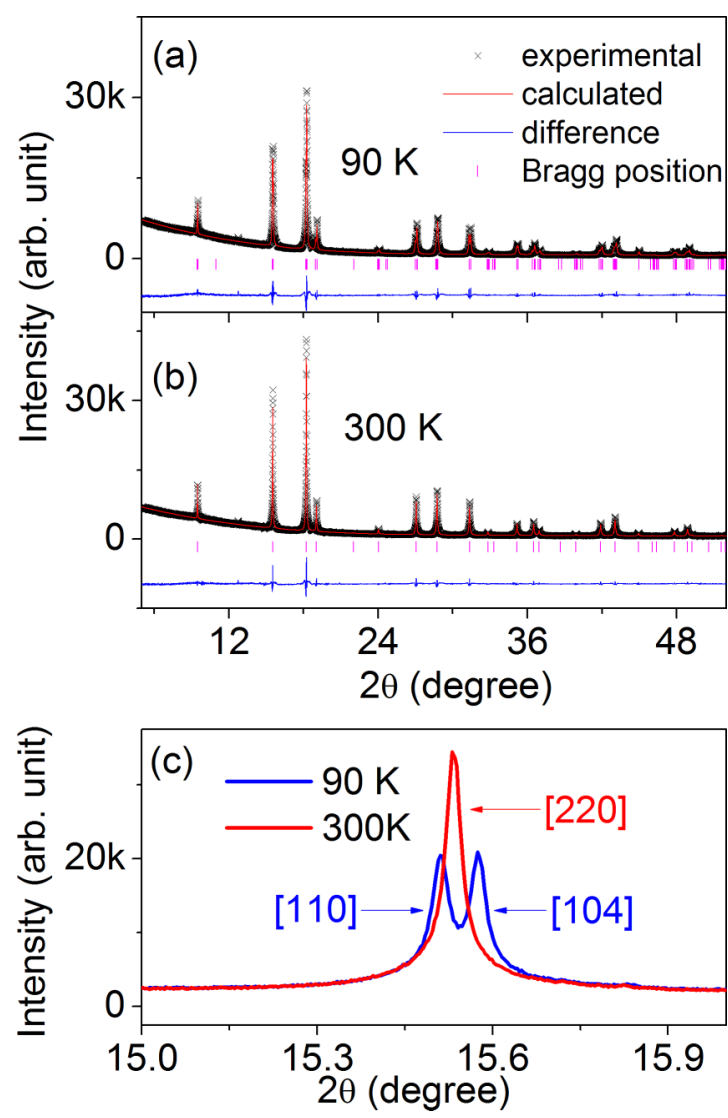

FIG. 10. X-ray diffraction patterns (synchrotron radiation) of $\mathrm{TbCo}_{2} \mathrm{Mn}_{0.1}$ at (a) $90 \mathrm{~K}$ and (b) $300 \mathrm{~K}$; (c) is a magnified plot showing the (cubic) [220] peak replaced by two rhombohedral peaks, [110] and [104], with cooling.

with second order transitions, we carried out an analysis of critical exponents using the Kouvel-Fisher (KF) method. The $\mathrm{KF}$ method can be described as follows [21]:

$$
\begin{gathered}
\frac{M_{S}(T)}{d M_{S}(T) / d T}=\frac{T-T_{C}}{\beta}, \\
\frac{\chi_{0}^{-1}(T)}{d \chi_{0}^{-1}(T) / d T}=\frac{T-T_{C}}{\gamma},
\end{gathered}
$$

where $M_{S}(T)$ and $\chi_{0}^{-1}(T)$ can be derived from the high field data of the Arrott plot as shown by the dashed lines in Fig. 5(a): the positive values of $x$ intercept and $y$ intercept of the dash line are the values of $\chi_{0}^{-1}(T)$ and $M^{2}{ }_{s}(T)$, respectively. Based on Eqs. (3) and (4), one can derive the corresponding

\begin{tabular}{|c|c|c|c|c|c|}
\hline \multicolumn{2}{|l|}{ Composition } & Technique & $\beta$ & $\gamma$ & $\delta$ \\
\hline $\mathrm{TbCo}_{2} \mathrm{Mn}_{x}$ & $x=0.1$ & Kouvel-Fisher method & $0.50 \pm 0.02$ & $1.01 \pm 0.03$ & $3.07 \pm 0.01$ \\
\hline & $x=0.2$ & & $0.49 \pm 0.02$ & $1.01 \pm 0.02$ & $3.20 \pm 0.02$ \\
\hline & $x=0.3$ & & $0.52 \pm 0.01$ & $1.01 \pm 0.02$ & $2.99 \pm 0.01$ \\
\hline 3D Hei & lodel & Theory & 0.365 & 1.386 & 4.80 \\
\hline Mean fie & heory & Theory & 0.500 & 1.000 & 3.00 \\
\hline $3 \mathrm{D} \mathrm{I}$ & & Theory & 0.325 & 1.241 & 4.82 \\
\hline
\end{tabular}

TABLE I. Deduced critical exponents $\beta, \gamma$, and $\delta$ for $\mathrm{TbCo}_{2} \mathrm{Mn}_{x}(x=0.1,0.2$, and 0.3$)$ compounds along with standard critical exponents of three standard theories: 3D Heisenberg model, mean field (MF) theory, and 3D Ising model. 

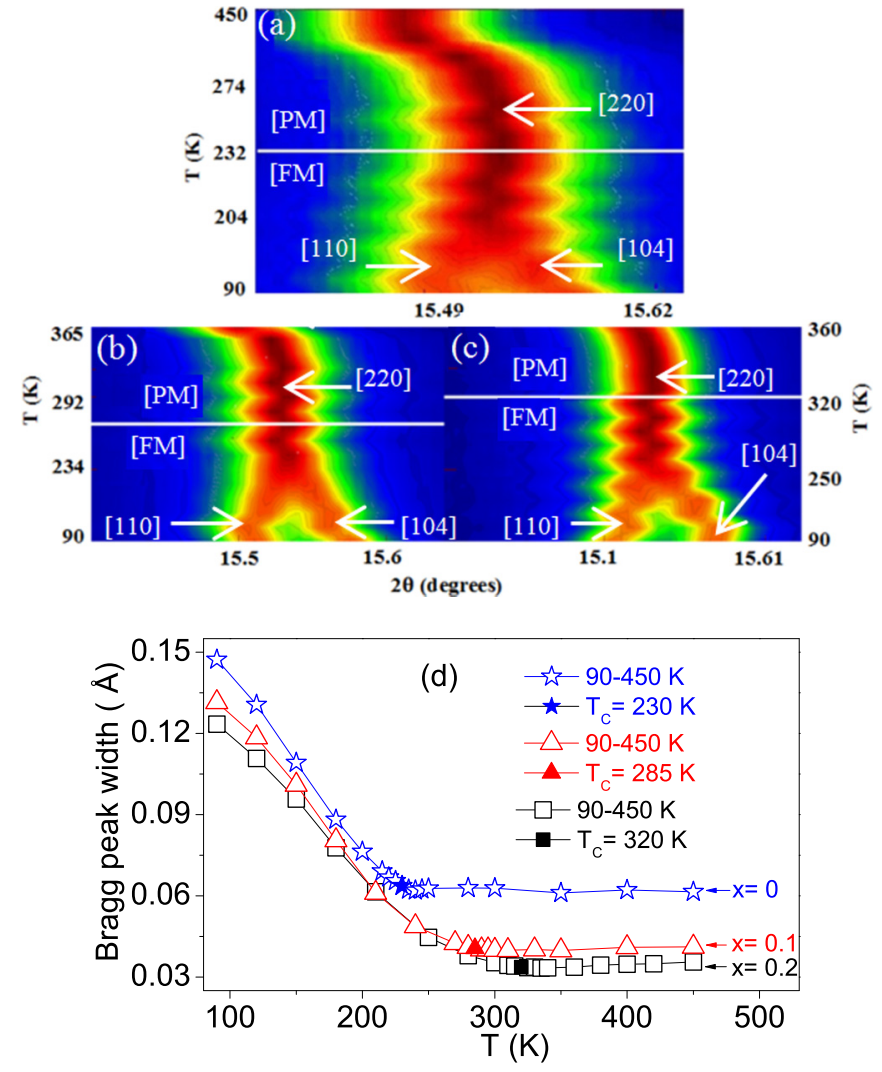

FIG. 11. Synchrotron $\mathrm{x}$-ray Bragg at various temperatures from $90 \mathrm{~K}$ to $450 \mathrm{~K}$ showing the [220] peak transforming into two small peaks ([110], [104]) after cooling to below $T_{C}$ for (a) $\mathrm{TbCo}_{2}$, (b) $\mathrm{TbCo}_{2} \mathrm{Mn}_{0.1}$, (c) $\mathrm{TbCo}_{2} \mathrm{Mn}_{0.2}$, and (d) combined peak widths (full width at half maximum) from $90 \mathrm{~K}$ to $450 \mathrm{~K}$.

values of slopes $1 / \beta, 1 / \gamma$, and $T_{C}$ using the linear fitting of $M_{S} /\left(d M_{S}(T) / d T\right)$ and $\chi_{0}^{-1}(T) /\left(d \chi_{0}^{-1}(T) / d T\right)$. The fitted $T_{C}$ values match well with the ones obtained from the $M-T$ curves in Fig. 2(a). Moreover, for all the samples the derived values are close to $\beta=0.5$ and $\gamma=1.0$ as shown in Fig. 6(a), Fig. 7(a), and Fig. 8(a).

The value of $\delta$ can be obtained by a linear fit to the higher field plots $\ln (\mathrm{M})$ vs $\ln (\mathrm{B})$ near $T_{C}$ as shown in the insets in Fig. 6(b), Fig. 7(b), and Fig. 8(b), according to the following equation [22]:

$$
M\left(B, T_{c}\right)=A_{0}(B)^{1 / \delta},
$$

where $A_{0}$ are the critical amplitudes. The values of $\delta$ for $\mathrm{TbCo}_{2} \mathrm{Mn}_{0.1}, \mathrm{TbCo}_{2} \mathrm{Mn}_{0.2}$, and $\mathrm{TbCo}_{2} \mathrm{Mn}_{0.3}$ are derived to be $3.07,3.20$, and 2.99 , respectively.

In addition, the value of $\delta$ can also be deduced using the Widom scaling relation $[8,20]$ :

$$
\delta=1+\gamma / \beta .
$$

All of the values derived for the critical exponents $\beta, \gamma$, and $\delta$ have been listed in Table I. One can conclude that for all the second order transitions of these three samples, the values of $\beta, \gamma$, and $\delta$ are close to $0.5,1.0$, and 3.0 within allowable experimental error, respectively. This indicates that the magnetic interactions in these compounds have long range order.

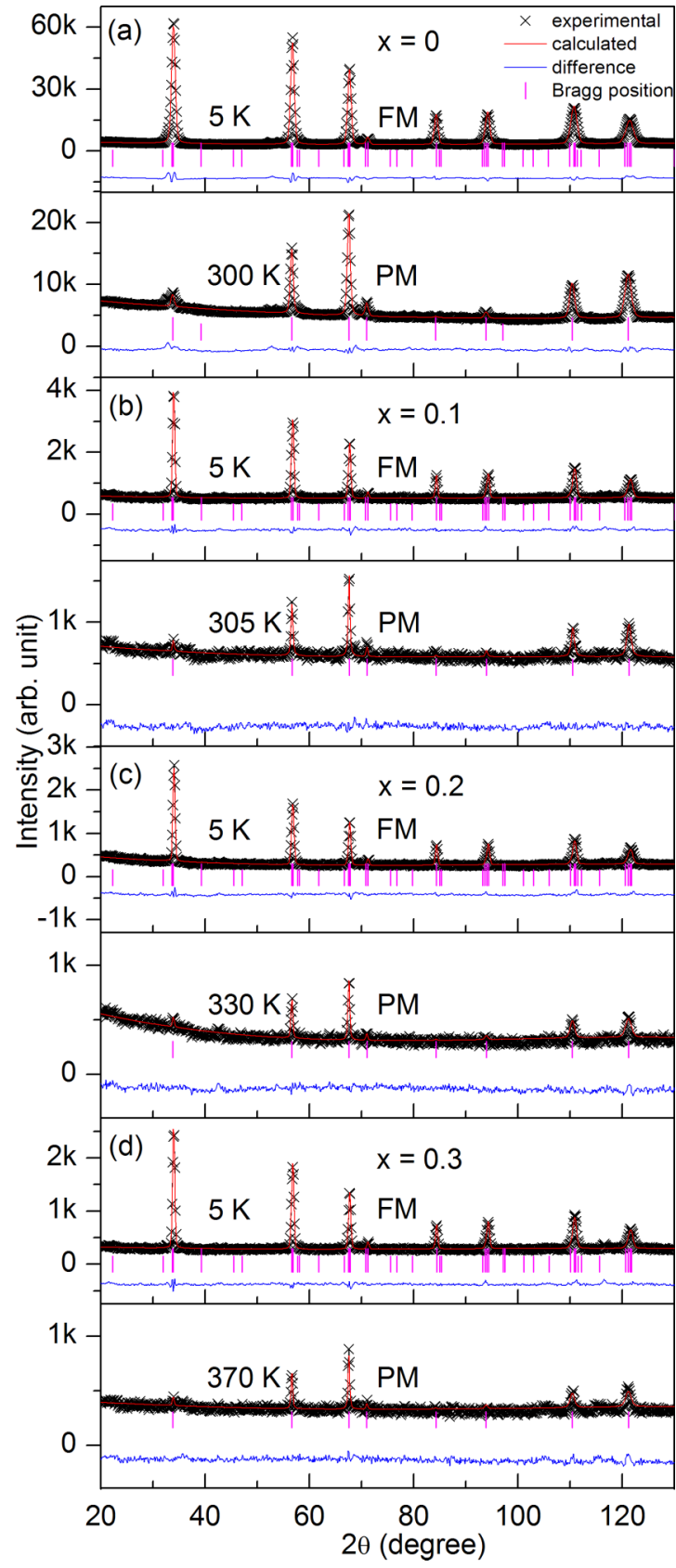

FIG. 12. Neutron-diffraction patterns of $\mathrm{TbCo}_{2} \mathrm{Mn}_{x}(x=0.1$, 0.2 , and 0.3 ) at $5 \mathrm{~K}$ (magnetic state) and higher temperature (paramagnetic state). The black crosses are the experimental neutrondiffraction data and the red lines represent the Rietveld refined pattern. The difference between the experimental and calculated patterns is shown at the bottom by the blue solid curves. The vertical bars indicate the position of allowed Bragg peaks.

Finally, using the scaling theory in the critical region, the reliability of exponents $\beta$ and $\gamma$ can be confirmed by the following equation [21]:

$$
M(H, \varepsilon)=\varepsilon^{\beta} f \pm\left(H / \varepsilon^{\beta+\gamma}\right),
$$

where $\varepsilon$ is the reduced temperature, $\left(T-T_{C}\right) / T_{C}$ and $f_{+}$ and $f_{-}$are regular analytical functions above and below $T_{C}$, respectively. The values of $\beta$ and $\gamma$ obtained through the 
TABLE II. Structure parameters and atomic moments of $\mathrm{TbCo}_{2} \mathrm{Mn}_{x}(x=0.1,0.2$, and 0.3$)$ compounds at $5 \mathrm{~K}$ and temperature near $T_{C}$. The uncertainties are shown for the $\mathrm{TbCo}_{2}$ data as an example.

\begin{tabular}{|c|c|c|c|c|c|c|c|c|}
\hline \multirow{2}{*}{$\begin{array}{l}\text { Parameters } \\
T(\mathrm{~K})\end{array}$} & \multicolumn{2}{|c|}{$x=0$} & \multicolumn{2}{|c|}{$x=0.1$} & \multicolumn{2}{|c|}{$x=0.2$} & \multicolumn{2}{|c|}{$x=0.3$} \\
\hline & 5 & 230 & 5 & 260 & 5 & 300 & 5 & 315 \\
\hline$a \times \sqrt{2}(\AA)$ & $7.176(1)$ & $7.195(5)$ & 7.177 & 7.192 & 7.173 & 7.198 & 7.167 & 7.191 \\
\hline$c / \sqrt{3}(\AA)$ & $7.223(4)$ & 7.197(1) & 7.193 & 7.194 & 7.195 & 7.198 & 7.189 & 7.192 \\
\hline$V /$ chemical formula $\left(\AA^{3}\right)$ & $46.50(3)$ & $46.57(9)$ & 46.31 & 46.51 & 46.27 & 46.59 & 46.15 & 46.49 \\
\hline $6 c\left(\mu_{B}\right)$ & $9.21(60)$ & $3.20(31)$ & 7.57 & 2.67 & 7.10 & 2.59 & 6.39 & 2.10 \\
\hline $3 b\left(\mu_{B}\right)$ & $1.60(30)$ & $0.81(21)$ & 1.50 & 1.14 & 1.84 & 0.69 & 1.51 & 0.80 \\
\hline $9 e\left(\mu_{B}\right)$ & $1.85(21)$ & $0.81(31)$ & 1.72 & 0.98 & 2.01 & 1.33 & 1.82 & 1.20 \\
\hline$M_{\text {total }}\left(\mu_{B}\right)$ & $5.76(61)$ & $1.58(31)$ & 4.35 & 0.55 & 3.25 & 0.57 & 3.06 & 0.00 \\
\hline
\end{tabular}

Kouvel-Fisher method [see Fig. 6(a), Fig. 7(a), and Fig. 8(a)] can then be used to obtain the $M / \varepsilon^{\beta}$ versus $H / \varepsilon^{(\beta+\gamma)}$ curves as shown in Fig. 6(c), Fig. 7(c), and Fig. 8(c). The resulting curves can be grouped in one of two clear types, one for temperatures above $T_{C}$ and the other one for temperatures below $T_{C}$. From the nature of the plots it can be concluded that all the calculated exponents $\beta$ and $\gamma$ and the corresponding $T_{C}$ are reliable for each sample.

\section{E. Heat capacity}

The temperature dependent heat capacity for $\mathrm{TbCo}_{2} \mathrm{Mn}_{0.1}$ at $B=0 \mathrm{~T}$ is shown in Fig. 9. An obvious peak appears at $284 \mathrm{~K}$ which is ascribed to the ferromagnetic transition of $\mathrm{TbCo}_{2} \mathrm{Mn}_{0.1}$. The total heat capacity $C(T)$ of a metallic magnetic material can be described as the sum of various contributions from phonons, electrons, and magnon [23]:

$$
C(T)=C_{\mathrm{ph}}(T)+C_{\mathrm{el}}(T)+C_{m}(T),
$$

where $C_{\mathrm{ph}}, C_{\mathrm{el}}$, and $C_{\mathrm{m}}$ are the lattice, electronic, and magnetic contributions, respectively. At lower temperature $(T \leqslant 10 \mathrm{~K})$ with the absence of a nearby magnetic phase transition, the heat capacity can be approximated as

$$
C(T) / T=\gamma+\beta T^{2},
$$

where $\gamma$ and $\beta$ are the electronic and phonon heat capacity coefficients, respectively. By applying a linear fitting approach to the low temperature specific heat at of $\mathrm{TbCo}_{2} \mathrm{Mn}_{0.1}$, one can obtain the values of $\gamma=30.5 \mathrm{~mJ} / \mathrm{mol} \mathrm{K}^{2}$ and $\beta=4.6 \times$ $10^{-4} \mathrm{~J} / \mathrm{mol} \mathrm{K}^{4}$. The electronic density of states $N\left(E_{F}\right)$ at the Fermi surface can be described by the formula [24]

$$
\gamma=\frac{k_{B}{ }^{2} \pi^{2}}{3} N\left(E_{F}\right),
$$

where $k_{B}$ is the Boltzmann constant. Using the value derived above, the $N\left(E_{F}\right)$ can be calculated to be 3.04 state/eV atom compared with 2.0 state/eV for similar material $\mathrm{TbNi}_{2}$ [25]. Similarly, the Debye temperature $\theta_{\mathrm{D}}$ can also be deduced from [23]

$$
\beta=\frac{12 \pi^{4} R}{5 \theta^{3} D} \cong \frac{1944 n}{\theta^{3}{ }_{D}},
$$

where $R$ is the universal gas constant and the number of atoms $n=3.1 \mathrm{f}$.u. The Debye temperature $\theta_{\mathrm{D}}$ for $\mathrm{TbCo}_{2} \mathrm{Mn}_{0.1}$ was derived to be $235 \mathrm{~K}$, which is close to the value reported for $\mathrm{TbNi}_{2} \mathrm{Mn}\left(\theta_{D}=200 \mathrm{~K}\right)$ [20].

\section{F. Magnetoelastic coupling: Synchrotron and neutron diffraction}

The $\mathrm{x}$-ray diffraction patterns (synchrotron radiation) along with the Rietveld refined results at $T=90 \mathrm{~K}$ (magnetic state) and $T=300 \mathrm{~K}$ (paramagnetic state) for $\mathrm{TbCo}_{2} \mathrm{Mn}_{0.1}$ are shown in Figs. 10(a) and 10(b), respectively. In this case of $\mathrm{TbCo}_{2} \mathrm{Mn}_{0.1}$ for synchrotron x-ray diffraction at $300 \mathrm{~K}$, the pattern factor, $R_{p}$, the weighted pattern factor, $R_{w p}$, and the expected pattern factor $R_{\exp }$ are found to be $5.41 \%, 6.79 \%$, and $2.66 \%$, respectively. For the pattern at $90 \mathrm{~K}$, the values of $R_{p}, R_{w p}$, and $R_{\exp }$ are 5.91, 7.54, and 2.97, respectively.

From refinements of XRD patterns at variable temperatures, we found for all the $\mathrm{TbCo}_{2} \mathrm{Mn}_{x}$ compounds that the structures exhibit a rhombohedral distortion below $T_{C}$. This transformation from the cubic to the rhombohedral structure is illustrated by a magnified plot in Fig. 10(c). It can be seen that the peak [220] at $300 \mathrm{~K}$ for the cubic structure $(F d \overline{3} m$

\begin{tabular}{|c|c|c|c|c|c|c|c|c|c|c|}
\hline \multirow[b]{3}{*}{$x$} & \multicolumn{4}{|c|}{ Cubic $F d \overline{3} m$} & \multicolumn{6}{|c|}{ Rhombohedral $R \overline{3} m$} \\
\hline & \multicolumn{2}{|c|}{$8 a$} & \multicolumn{2}{|c|}{$16 d$} & \multicolumn{2}{|c|}{$6 c$} & \multicolumn{2}{|c|}{$3 b$} & \multicolumn{2}{|c|}{$9 e$} \\
\hline & $\mathrm{Tb}$ & $\mathrm{Mn}$ & Co & $\mathrm{Mn}$ & $\mathrm{Tb}$ & $\mathrm{Mn}$ & Co & $\mathrm{Mn}$ & $\mathrm{Co}$ & $\mathrm{Mn}$ \\
\hline 0 & $100.00 \%$ & $0.00 \%$ & $100.00 \%$ & $0.00 \%$ & $100.00 \%$ & $0.00 \%$ & $100.00 \%$ & $0.00 \%$ & $100.00 \%$ & $0.00 \%$ \\
\hline 0.1 & $96.77 \%$ & $3.23 \%$ & $96.77 \%$ & $3.23 \%$ & $96.77 \%$ & $3.23 \%$ & $96.77 \%$ & $3.23 \%$ & $96.77 \%$ & $3.23 \%$ \\
\hline 0.2 & $93.76 \%$ & $6.24 \%$ & $93.76 \%$ & $6.24 \%$ & $93.76 \%$ & $6.24 \%$ & $93.76 \%$ & $6.24 \%$ & $93.76 \%$ & $6.24 \%$ \\
\hline 0.3 & $90.93 \%$ & $9.07 \%$ & $90.93 \%$ & $9.07 \%$ & $90.93 \%$ & $9.07 \%$ & $90.93 \%$ & $9.07 \%$ & $90.93 \%$ & $9.07 \%$ \\
\hline
\end{tabular}
space group) is replaced by two peaks [110] and [104] of the rhombohedral structure (space group $R \overline{3} \mathrm{~m}$ ) at $90 \mathrm{~K}$. Many

TABLE III. Atomic (Tb, Co, and Mn) occupancy in cubic and rhombohedral structure, respectively. 


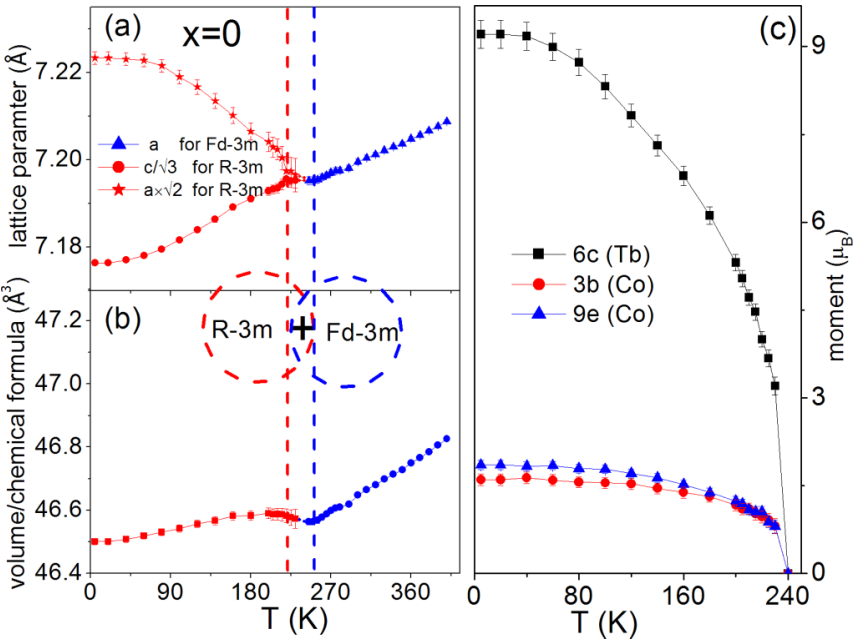

FIG. 13. Temperature dependence in $\mathrm{TbCo}_{2}$ of (a) lattice parameters, (b) volume per chemical formula, and (c) ordered magnetic moment in each site $(6 c, 3 b$, and $16 d)$. Between the two vertical dashed lines is the phase transition region with possible both rhombohedral and cubic structure apparently present.

details of the structural features around $T_{C}$ can be observed from our high resolution x-ray powder diffraction study. For example, the temperature dependences of the synchrotron diffraction patterns near the $R T$ cubic [220] peak are shown in Figs. 11(a), 11(b), and 11(c). It can be seen that below the Curie temperature $T_{C}$ (marked by the white horizontal line), the [220] peak transforms into two smaller separated peaks as the temperature is lowered following a period of distortion. The peak transformation can be observed more clearly in the plot of temperature dependent peak widths shown in Fig. 11(d). The solid symbols within each plot mark the Curie temperature for $\mathrm{TbCo}_{2}, \mathrm{TbCo}_{2} \mathrm{Mn}_{0.1}$, and $\mathrm{TbCo}_{2} \mathrm{Mn}_{0.2}$, respectively, and also the onset of the [220]

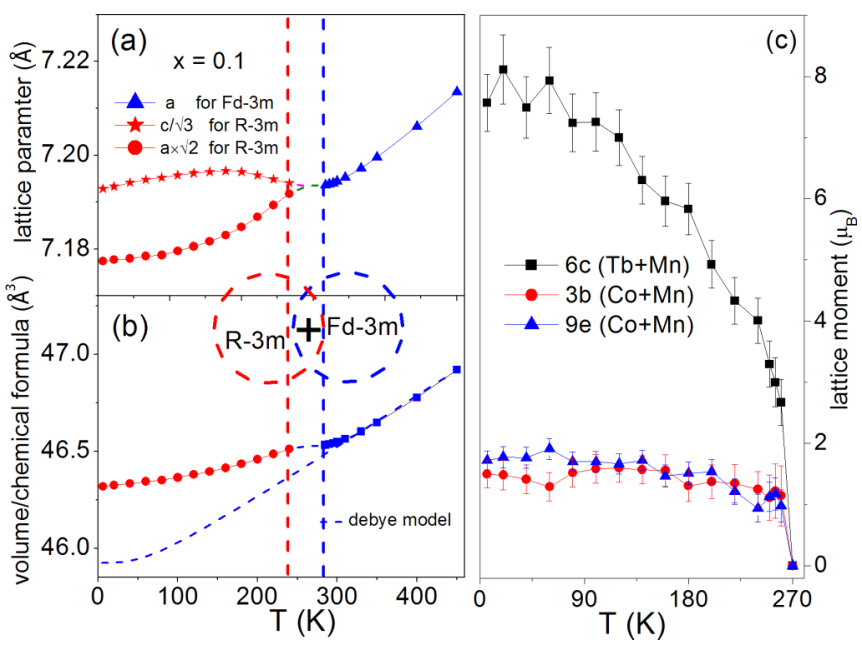

FIG. 14. Temperature dependence for $\mathrm{TbCo}_{2} \mathrm{Mn}_{0.1}$ of (a) lattice parameters, (b) volume per chemical formula, and (c) ordered magnetic moment in each site $(6 c, 3 b$, and $16 d)$. Between the two vertical dashed lines is the phase transition region with possible both rhombohedral and cubic structure apparently present.

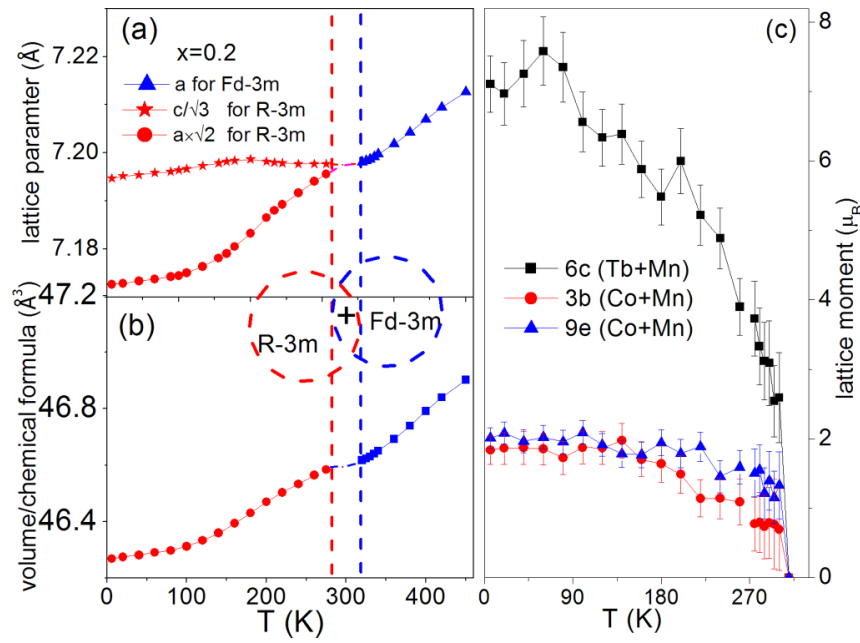

FIG. 15. Temperature dependence for $\mathrm{TbCo}_{2} \mathrm{Mn}_{0.2}$ of (a) lattice parameters, (b) volume per chemical formula, and (c) ordered magnetic moment of each site $(6 c, 3 b$, and $16 d)$. Between the two vertical dashed lines is the phase transition region with possible both rhombohedral and cubic structure apparently present.

peak distortion with further cooling. When the peak width nominally assigned to [220] is observed to become much broader than that above $T_{C}$, it reflects that the peak has transformed into the two new peaks as shown in Figs. 11(a), 11(b), and 11(c). Clearly we can conclude that there is a structural transition, cubic to rhombohedral, concurrent with the magnetic state change from paramagnetic to ferromagnetic. However, it cannot be definitively determined from current data whether this transformation proceeds via a rhombohedral distortion process or a period of phase coexistence.

In order to determine the magnetic structure and derive magnetic information, we carried out a variable temperature neutron diffraction study ( $5 \mathrm{~K}$ to $400 \mathrm{~K}$ ). This neutron diffrac-

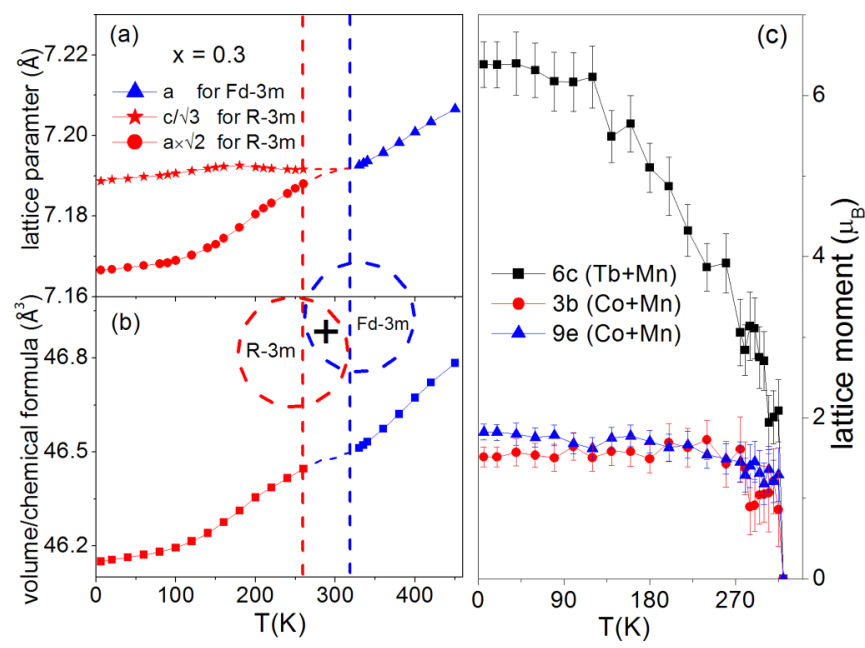

FIG. 16. Temperature dependence for $\mathrm{TbCo}_{2} \mathrm{Mn}_{0.3}$ of (a) lattice parameters, (b) volume per chemical formula, and (c) ordered magnetic moment in each site $(6 c, 3 b$, and $16 d)$. Between the two vertical dashed lines is the phase transition region with possible both rhombohedral and cubic structure apparently present. 
tion data, including refinements carried out with the Fullprof package, are shown in Fig. 12 to Fig. 16 for $x=0,0.1,0.2$, and 0.3 , respectively, and main results are listed in Table II. The results of the atomic occupancies ( $\mathrm{Tb}, \mathrm{Co}$, and $\mathrm{Mn}$ ) in both the cubic and rhombohedral structures estimated via the refinements are listed in Table III. In order to compare directly between the lattice parameters of the cubic structure $(F d \overline{3} m)$ and the rhombohedral structure $(R \overline{3} m)$ at the transition, we modify the rhombohedral lattice parameters by using $a \times \sqrt{2}$ and $c / \sqrt{3}$. The results for $x=0$ are shown in Fig 13. The variation in lattice parameters $(a \times \sqrt{2}$ and $c / \sqrt{3})$, with temperature below $T_{C}$ display strong anisotropy: the lattice parameter $a \times$ $\sqrt{2}$ (red solid circles) increases monotonically while $c / \sqrt{3}$ (red solid stars) decreases with increasing temperature. At $T_{C}$ the crystal structure transforms from rhombohedral to cubic above which $a$ increases monotonically with temperature. The temperature dependence of the cell volume [Fig. 13(b)] exhibits behavior typical of a first order transition with a noticeable drop near $T_{C}$. In contrast with $\mathrm{TbCo}_{2}$, the temperature variation of the lattice parameter $a \times \sqrt{2}$ in the Mn doped compounds tends to become less pronounced but $c / \sqrt{3}$ still increases monotonically. The combined effect therefore is a more pronounced change in volume in the Mn doped cases. In addition a theoretical estimate based on Debye theory and using the Debye temperature $\theta_{\mathrm{D}}$ obtained from data of Fig. 9 (dash lines) is also plotted for the $x=0.1$ case in Fig. 14(b). The spontaneous volume magnetostriction $\omega_{\mathrm{s}}\left(=\Delta V_{m} / V\right)$ at $5 \mathrm{~K}$ for $x=0.1$ is calculated to be $8.5 \times 10^{-3}$. In our refinement, it is assumed that the moments of the magnetic atoms in the compound have a collinear alignment similar to the model described in the investigation for $\mathrm{TbCo}_{2}$ [26]. The $\mathrm{Tb}$ moment is derived to be $9.21 \mu_{\mathrm{B}}$ at $5 \mathrm{~K}$, while the Co moments at the $3 b$ site and the $9 e$ site are $1.85 \mu_{\mathrm{B}}$ and $1.60 \mu_{\mathrm{B}}$, respectively. Since the limitation of data resolution would not support the increased number of refinement parameters for the compounds containing Mn, we derive only the average moment for each site as shown in Figs. 14-16.

As an example, the parameters resulting from refinement of the neutron diffraction patterns at $5 \mathrm{~K}$ and near $T_{C}$ for all the $\mathrm{TbCo}_{2} \mathrm{Mn}_{x}$ compounds are listed in Table II. It can be seen that the average moment at the rare earth site decreases with increasing Mn content, which indicates that some Mn atoms substitute onto this site and carry a magnetic moment that couples antiferromagnetically with the $\mathrm{Tb}$.

\section{CONCLUSIONS}

Compounds $\mathrm{TbCo}_{2} \mathrm{Mn}_{x}(x=0.1,0.2$, and 0.3$)$ with cubic $\mathrm{MgCu}_{2}$-type structure at $300 \mathrm{~K}$ have been successfully synthesized. Our study of the magnetic properties in the series indicates that magnetic phase transition for the $\mathrm{TbCo}_{2}$ compound is first order, but the addition of Mn changes the nature of the transition to second order. Furthermore, the addition of Mn into $\mathrm{TbCo}_{2}$ compounds leads to a significant increase in $T_{C}$ but an obvious reduction of spontaneous magnetization. A detailed analysis of critical exponent of the magnetic behavior around $T_{C}$ shows that these systems indeed undergo a second order phase transition and that the magnetic exchange interaction is long range. Our high resolution x-ray powder diffraction study confirms that all compounds crystallize in the cubic Laves phase $C 15$ structure above their Curie temperature $T_{C}$ and transform to a rhombohedral phase (space group $R \overline{3} \mathrm{~m}$ ) below $T_{C}$. Magnetic structure and magnetic moments below $T_{C}$ have been determined through a variable temperature neutron diffraction investigation.

\section{ACKNOWLEDGMENTS}

The work is supported in part by grants from the Australian Research Council, Australian Institute of Nuclear Science and Engineering, and Australian Synchrotron.
[1] R. Nirmala, Ya. Mudryk, V. K. Pecharsky, and K. A. Gschneidner, Phys. Rev. B 76, 014407 (2007).

[2] B. Maji, K.G. Suresh, and A. K. Nigam, J. Magn. Magn. Mater. 322, 2415 (2010).

[3] C. W. Zhang, Z. Zhang, S. Q. Wang, H. Li, J. M. Dong, N. S. Xing, Y. Q. Guo, and W. Li, Solid State Commun. 142, 477 (2007).

[4] J. L. Wang, M. F. Md Din, S. J. Kennedy, F. Hong, S. J. Campbell, A. J. Studer, G. H. Wu, Z. X. Cheng, and S. X. Dou, J. Appl. Phys. 115, 17E135 (2014).

[5] J. L. Wang, C. C. Tang, G. H. Wu, Q. L. Liu, N. Tang, W. Q. Wang, W. H. Wang, F. M. Yang, J. K. Liang, F. R. de Boer, and K. H. J. Buschow, Solid State Commun. 121, 615 (2002).

[6] J. L. Wang, C. Marquina, M. R. Ibarra, and G. H. Wu, Phys. Rev. B 73, 094436 (2006).

[7] D. D. Jackson, S. K. McCall, S. T. Weir, A. B. Karki, D. P. Young, W. Qiu, and Y. K. Vohra, Phys. Rev. B 75, 224422 (2007).

[8] M. Halder, S. M. Yusu, M. D. Mukadam, and K. Shashikala, Phys. Rev. B 81, 174402 (2010).
[9] J. L. Wang, S. J. Campbell, M. Hofmann, M. Hoelzel, R. Zeng, S. X. Dou, and S. J. Kennedy, J. Appl. Phys. 111, 07E334 (2012).

[10] E. G. Gerasimov, N. V. Mushnikov, P. B. Terentev, V. S. Gaviko, and A. A. Inishev, J. Alloys Compd. 571, 132 (2013).

[11] E. G. Gerasimov, N. V. Mushnikov, A. A. Inishev, P. B. Terentev, and V. S. Gaviko, J. Alloys Compd. 680, 359 (2016).

[12] D. Bloch, D. M. Edwards, M. Shimizu, and J. Voiron, J. Phys. F: Met. Phys. 5, 1217 (1975).

[13] E. Gratzi, H. Sassikt, and H. Nowotnyx, J. Phys. F: Met. Phys. 11, 429 (1981).

[14] N. H. Duc, D. T. Kim Anh, and P. E. Brommer, Physica B 319, 1 (2002).

[15] B. K. Banerjee, Phys. Lett. 12, 16 (1964).

[16] C. M. Bonilla, F. Bartolomé, L. M.García, M. Parra-Borderías, J. Herrero-Albillos, and V. Franco, J. Appl. Phys. 107, 09E131 (2010).

[17] M. Parra-Borderias, F. Bartolome, J. Herrero-Albillos, and L. M. Garcia, J. Alloys Compd. 481, 48 (2009). 
[18] C. M. Bonilla, J. Herrero-Albillos, F. Bartolomé, L. M. García, M. Parra-Borderías, and V. Franco, Phys. Rev. B 81, 224424 (2010).

[19] Q. Y. Ren, W. D. Hutchison, J. L. Wang, A. J. Studer, and S. J. Campbell, J. Alloys Compd. 693, 32 (2017).

[20] J. L. Wang, S. J. Campbell, S. J. Kennedy, R. Zeng, S. X. Dou, and G. H. Wu, J. Phys.: Condens. Matter 23, 216002 (2011).

[21] P. Shambal, J. L. Wang, J. C. Debnathl, S. J. Kennedy, R. Zeng, M. F. Md Din, F. Hong, Z. X. Cheng, A. J. Studer, and S. X. Dou, J. Phys.: Condens. Matter 25, 056001 (2013).
[22] N. Khan, A. Midya, K. Mydeen, P. Mandal, A. Loidl, and D. Prabhakaran, Phys. Rev. B 82, 064422 (2010).

[23] P. Kumar, K. G. Suresh, A. K. Nigam, and S. K. Malik, J. Appl. Phys. 103, 013909 (2008).

[24] E. G. Gerasimov, T. Kanomata, and V. S. Gaviko, Physica B 390, 118 (2007).

[25] M. A. Korotin, N. A. Skorikov, A. V. Efremov, and A. O. Shorikov, J. Magn. Magn. Mater. 397, 115 (2016).

[26] Z. W. Ouyang, F. W. Wang, Q. Hang, W. F. Liu, G. Y. Liu, J. W. Lynn, J. K. Liang, and G. H. Rao, J. Alloys Compd. 390, $21(2005)$ 